\title{
Efectos del disturbio humano sobre la dinámica poblacional de Ocypode quadrata (Decapoda: Ocypodidae) en playas del Caribe mexicano
}

\author{
Frank A. Ocaña ${ }^{1}$, Alberto de Jesús-Navarrete ${ }^{2}$, Rosa M. de Jesús-Carrillo ${ }^{2}$ \& \\ José J. Oliva-Rivera ${ }^{2}$ \\ 1. Programa de doctorado en Ecología y Desarrollo Sustentable, El Colegio de la Frontera Sur, Chetumal, Quintana Roo, \\ México; frankocisat@gmail.com, focana@ecosur.edu.mx \\ 2. Departamento de Sistemática y Ecología Acuática, El Colegio de la Frontera Sur, Chetumal, Quintana Roo, México; \\ anavarre@ecosur.mx, rosycoquina@gmail.com, joliva@ecosur.mx
}

Recibido 30-VI-2015. Corregido 25-IV-2016. Aceptado 26-V-2016.

\begin{abstract}
Effects of human disturbance on the population dynamics of Ocypode quadrata (Decapoda: Ocypodidae) in beaches of the Mexican Caribbean. The ghost crab Ocypode quadrata is a relevant species in the Western Atlantic beaches, and has been proposed as an indicator of human impact in these ecosystems. Eventhough some studies have covered various aspects of its natural history, no evaluations on the effects of human disturbance on its population dynamics have been made. This work compared the abundance, growth, and mortality of $O$. quadrata in two disturbed beaches (Aventuras and Majahual) and two preserved beaches (Xcacel and Puerto Angel) from the Mexican Caribbean. For this, nine samplings were made on each beach (every two to three months) between March 2013 and November 2014. Crabs were collected manually by three people, during the night first hours and for one hour, and a total area of $9000 \mathrm{~m}^{2}$ per beach. The crabs were measured, weighed, and separated into three groups: juvenile, male and female; while abundance was estimated by the number of collected crabs, and growth parameters were estimated using length frequencies. A total of 1047 crabs were sampled in the four beaches; a higher crab abundance was found in the preserved beaches, but significant differences were only found between Puerto Angel and Majahual (Tukey, $\mathrm{p}<0.05$ ). The adultjuvenile ratio was 1:1 in Xcacel and Aventuras, while in Majahual, more juveniles than adults were collected; and Puerto Angel had more adults than the juveniles. The size structure at Xcacel ranged from 4-32 mm, but in the rest of the beaches the minimum size was $5 \mathrm{~mm}$. The maximum size at Aventuras, Majahual and Puerto Angel were $27 \mathrm{~mm}, 25 \mathrm{~mm}$ and $30 \mathrm{~mm}$, respectively. The modal progression analysis of length's pooled data, revealed the presence of at least two groups of ages in all beaches. For all beaches, individuals smaller than 10 $\mathrm{mm}$ length were collected in almost all sampling months, indicating a continuous recruitment throughout the year. The length-weight relationship of the ghost crab was found isometric in the two preserved beaches, and negative allometric at the two impacted beaches. Crabs from Puerto Angel showed the highest body condition (Tukey, $\mathrm{p}<0.05$ ). K-values ranged from 0.6-0.97/year and $\mathrm{L} \infty$ from $29.0 \mathrm{~mm}$ to $33.6 \mathrm{~mm}$ with higher values for the two preserved beaches. The highest mortality values were found in Xcacel and Aventuras. In the disturbed beaches, the destruction of the dunes, the mechanical cleaning and the removal of organic matter, seem to be the main causes that lead to a low abundance of crabs, their slower growth and higher mortalities. However, in the best preserved beaches, biological interactions appear to be the most important aspects in regulating the population dynamics of this species. These results supported the information that the ghost crab is a good indicator of human disturbance, and its population changes were clearly measurable and comparable among protected and impacted beaches; these results are also relevant for the management of sandy beaches in the Mexican Caribbean. Rev. Biol. Trop. 64 (4): 1625-1641. Epub 2016 December 01.
\end{abstract}

Key words: ghost crab, sandy beaches, biological indicator, growth, mortality. 
Las poblaciones y comunidades de invertebrados de las playas arenosas están reguladas, fundamentalmente, por un ambiente muy dinámico (Defeo \& McLachlan, 2005). Por otra parte, las respuestas de los organismos dependen de su historia natural, de tal forma que los invertebrados de la zona intermareal pueden presentar diferentes modos de vida que aquellos que viven en la zona supralitoral (Defeo \& McLachlan, 2011). Las especies que habitan en la zona supralitoral de las playas arenosas presentan cinco adaptaciones claves para su supervivencia: movilidad, construcción de galerías, ritmos circadianos, orientación y algún nivel de plasticidad en su comportamiento (Nel et al., 2014; Scapini, 2014).

La zona supralitoral de las playas arenosas puede sufrir varias transformaciones debidas a los disturbios ocasionados por el hombre. Estos disturbios pueden, por tanto, tener un fuerte impacto sobre los organismos que habitan esta zona. En este sentido, se ha demostrado que la fuerte presión ejercida por la urbanización y el desarrollo turístico en las playas, ha llevado a la extinción local de especies (Hubbard, Dugan, Schooler, \& Viola, 2014).

Entre los organismos más conspicuos de la zona supralitoral de las playas arenosas tropicales y subtropicales, se encuentran los cangrejos fantasma (Ocypode spp.) (Lucrezi \& Schlacher, 2014). El género Ocypode abarca 21 especies a nivel mundial, donde Ocypode quadrata Fabricius 1787 es la única presente en el Atlántico occidental (Sakai \& Türkay, 2013). El registro fósil data desde el Pleistoceno tardío (Portell, Turner, \& Beerensson, 2003) y su distribución actual es desde Rhode Island en Estados Unidos hasta Río Grande del Sur en Brasil (Melo, 1996). Aunque sus poblaciones pueden ocupar diferentes tipos de playas con diferentes niveles de impacto humano, su distribución puede estar limitada por la temperatura y la salinidad (Williams, 1984; Rosa \& Borzone, 2008).

Hasta el momento los estudios sobre esta especie se han enfocado en su biología reproductiva, crecimiento y morfología (Haley, 1969, 1972; Díaz \& Costlow, 1972; Negreiros-Fransozo, Fransozo, \& Bertini, 2002;
Antunes et al., 2010), en estudios fisiológicos (Santos \& Moreira, 1999; Vinagre, Amaral, Ribarcki, Silveira, \& Périco, 2007; Tullis \& Andrus, 2011) y ritmo de actividad (Valero-Pacheco, Álvarez, Abarca-Arenas, \& Escobar, 2007).

$O$. quadrata tiene un amplio espectro de alimentación, siendo detritívoro, carroñero (Robertson \& Pfeiffer, 1982; Branco, Hillesheim, Fracasso, Christoffersen, \& Evangelista, 2010), y un activo predador sobre anfípodos (Morrow, Bell, \& Tewfik, 2014) y neonatos de tortugas (Barton \& Roth, 2008). Constituye una presa importante para mamíferos (e.g. Procyon lotor) (Barton \& Roth, 2008) y aves nocturnas (Blankensteyn, 2006; Branco et al., 2010). Por lo tanto, O. quadrata tiene un papel muy importante en la transferencia de energía en diferentes niveles tróficos y constituye un depredador tope en playas arenosas (Wolcott, 1978; Morrow et al., 2014).

Los estudios sobre dinámica poblacional de invertebrados de playas arenosas, involucran la medición de variables como la abundancia, el crecimiento, la estructura de tallas, la reproducción y la mortalidad (McLachlan \& Brown, 2006). Algunas de estas variables han sido tratadas por separado en estudios poblacionales de $O$. quadrata. Un estudio reciente (Pombo, 2015) aportó datos sobre el crecimiento de esta especie; sin embargo, la estimación de los parámetros de crecimiento se basó en un método indirecto, y ha tomado en cuenta el diámetro de las galerías, lo que, a consideración de esta autora, podría implicar un sesgo en la estimación. Por otra parte, a excepción de los trabajos realizados por Araújo, Rosa y Fernandes (2008), Valero-Pacheco et al. (2007) y por Ocaña, Vega y Córdova (2012), la mayoría de las investigaciones sobre esta especie se han llevado a cabo hacia los extremos de su distribución. Teniendo en cuenta que esta especie es usada como un indicador del impacto humano en las playas arenosas, el objetivo del presente trabajo fue determinar el efecto que tiene el disturbio humano sobre la dinámica poblacional de O. quadrata en playas del Caribe mexicano. Por tanto, se plantea la hipotésis 
de que en las playas impactadas, el cangrejo fantasma presenta menor abundancia, mayor mortalidad y menor crecimiento que en las playas conservadas, debido a la acción conjunta de la limpieza de playa, el pisoteo continuo y la afectación de las dunas.

\section{MATERIALES Y MÉTODOS}

Área de estudio: Quintana Roo se encuentra hacia el este de la Península de Yucatán, México. El litoral de Quintana Roo, está compuesto fundamentalmente por playas arenosas y una pequeña parte de costa rocosa sin acantilados (Tello-Taracena \& Castellanos-Martínez,
2011). El estado se encuentra en una región frecuentada por el paso de los huracanes; el clima es cálido subhúmedo con una temperatura media anual de $25.5^{\circ} \mathrm{C}$ y una precipitación promedio de $1504.3 \mathrm{~mm}$ al año (HerreraSansores, 2011). En esta región se diferencian tres épocas climáticas: lluvias (junio-octubre), nortes o frentes fríos (noviembre-febrero) y secas (marzo-mayo). Las mareas son mixtas semidiurnas con una variación promedio inferior a $0.5 \mathrm{~m}$ (Carrillo, Palacios-Hernández, Yescas, \& Ramírez-Manguilar, 2009).

Para este estudio fueron seleccionadas cuatro playas del estado de Quintana Roo (Fig. 1): Aventuras $\left(20^{\circ} 21^{\prime} \mathrm{N}-8^{\circ} 19^{\prime} \mathrm{W}\right)$,

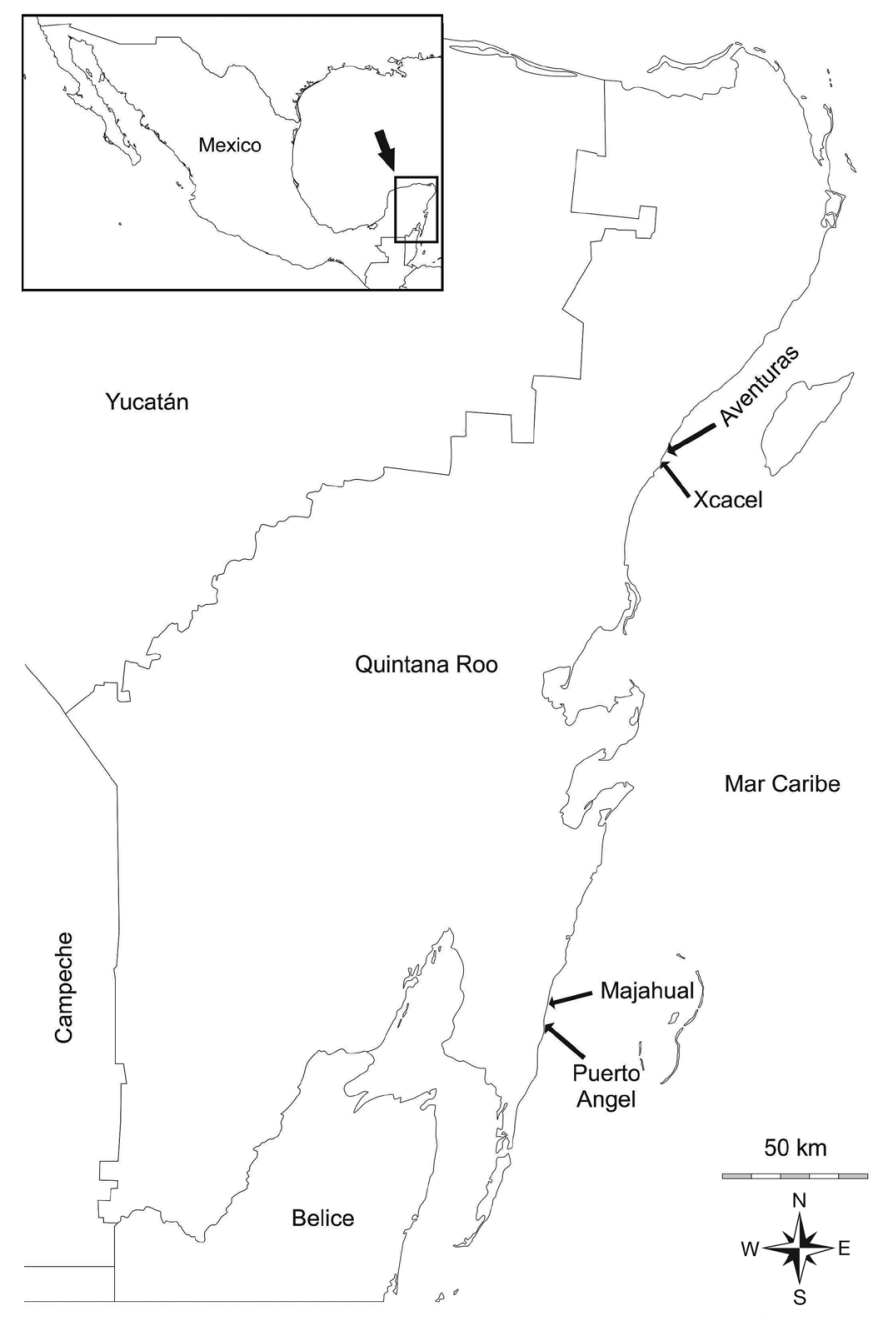

Fig. 1. Ubicación de los sitios de estudio. / Fig. 1. Location of the study sites. 
Xcacel $\left(20^{\circ} 20^{\prime} \mathrm{N}-87^{\circ} 20^{\prime} \mathrm{W}\right)$, Majahual $\left(18^{\circ} 42^{\prime} \mathrm{N}-87^{\circ} 42^{\prime} \mathrm{W}\right)$ y Puerto Ángel (18 $36^{\circ}$ ' $\left.\mathrm{N}-87^{\circ} 44^{\prime} \mathrm{W}\right)$. Aunque no se pudieron medir todos los indicadores propuestos por McLachlan, Defeo, Jaramillo, y Short (2013) para la categorización del potencial de conservación y recreativo, existen elementos para diferenciar a las playas de acuerdo al nivel de disturbio. Aventuras y Majahual son playas altamente impactadas con facilidades turísticas que cubren gran parte de la zona de dunas, y en la segunda esta zona fue destruida debido a la presencia de hoteles, casas y pequeños comercios. En ambas playas se realizan labores diarias de limpieza manual, y en Aventuras muchas veces se usa un vehículo motorizado para recoger los restos de macrófitos y materia orgánica arrojada por el mar. Xcacel y Puerto Ángel pueden considerarse como playas más conservadas debido a que las dunas presentan una alta cobertura vegetal, no existen infraestructuras para la recreación y en ambas playas anidan tortugas marinas. Por tanto, en una escala nominal el grado de conservación de las playas es mayor en Xcacel y Puerto Ángel que en Aventuras y Majahual.

Aunque todas las playas están, en cierta forma, protegidas por un sistema arrecifal, algunas presentan mayor influencia del oleaje que otras. Aventuras está protegida, en una parte de la playa, por un rompeolas que disminuye la acción del oleaje. Majahual es una playa dentro de una laguna arrecifal muy somera $(0.5 \mathrm{~m})$ con muy poca influencia del oleaje, además presenta un muelle de concreto en un extremo; mientras, Xcacel y Puerto Ángel son playas más expuestas al oleaje.

Variables abióticas: Se realizaron nueve muestreos en cada playa entre marzo 2013 y noviembre 2014, con intervalos entre dos y tres meses. A lo largo de cada playa, se trazaron seis transectas perpendiculares desde el nivel inferior de marea hasta el límite superior de la playa (borde de la duna o de infraestructuras). La disposición de las transectas se realizó al azar en pares localizadas a $30 \mathrm{~m}$ de distancia y una separación entre pares de aproximadamente $300 \mathrm{~m}$, para contemplar diferentes escalas espaciales. En cada transecta se determinó la pendiente con el uso de un clinómetro y la amplitud del perfil con el uso de una cinta métrica. También se determinó el ancho de la zona de resaca, midiendo la amplitud de cinco olas consecutivas desde el nivel inferior de marea, hasta el nivel superior de la resaca de la ola sobre la cara de la playa. En tres muestreos (uno en cada época climática) se determinó la altura $(\mathrm{cm})$ y periodo de la ola (s) en tres puntos fijos separados a $300 \mathrm{~m}$, a lo largo de la playa. En cada punto se midió la diferencia de altura de diez olas consecutivas usando una vara graduada en centímetros; posteriormente se midió el periodo de diez olas consecutivas usando un cronómetro (adaptado de Herrmann et al., 2009).

En dos ocasiones se tomaron nueve muestras de sedimento $(500 \mathrm{~g})$ en cada playa, ocupando la cara de la playa, la berma y la duna. En el laboratorio se determinó el tamaño medio del sedimento $\left(\mathrm{Md}_{50}, \mathrm{~mm}\right)$ y su contenido de materia orgánica (MOT, \%) de acuerdo a los métodos descritos por Buchanan (1984). El estado morfodinámico de las playas se determinó a través del parámetro de Dean $(\Omega)$ mediante la ecuación $\Omega=\mathrm{A}_{\mathrm{o}} / \mathrm{V}_{\mathrm{s}} \mathrm{T}$; donde $\mathrm{A}_{\mathrm{o}}$ es la altura de la ola $(\mathrm{cm}), \mathrm{V}_{\mathrm{s}}$ es la velocidad de caída del sedimento $(\mathrm{cm} / \mathrm{s})$ y $\mathrm{T}$ es el periodo de la ola (s) (Short, 1996). $\mathrm{V}_{\mathrm{s}}$ se determinó a partir de $\mathrm{Md}_{50}$, usando las tablas de Gibbs, Matthews, y Link (1971).

Para comparar el ancho, la pendiente, la amplitud de resaca y el contenido de materia orgánica entre las playas, se realizó un análisis de varianza de una vía (ANOVA) con los datos acumulados de todos los muestreos. Para cumplir con los requisitos de normalidad y homogeneidad de varianza, los datos originales fueron transformados a raíz cuadrada cuando fue necesario; en el caso del contenido de materia orgánica se realizó una transformación arcoseno. En el caso de detectarse diferencias significativas $(\mathrm{p}<0.05)$, se realizó una prueba de Tukey a posteriori (Zar, 1999).

Muestreo y abundancia: Se realizaron nueve muestreos en cada playa en el mismo periodo; durante diciembre de 2013 no se pudo 
realizar colecta de individuos debido a condiciones adversas del tiempo. Los muestreos se realizaron en las dos primeras horas de la noche; este criterio fue tomado de ValeroPacheco et al. (2007) quienes reportan que durante estas horas los cangrejos son más activos. Para la abundancia total por playa, cada muestreo se realizó en un área de $600 \mathrm{~m}$ de largo con un ancho aproximado de $15 \mathrm{~m}$, entre dos puntos fijos que sirvieron de referencia, totalizando $9000 \mathrm{~m}^{2}$. Tres personas recorrieron el área de muestreo de un extremo a otro durante una hora, auxiliadas de lámparas de luz blanca. La primera reacción de los cangrejos ante el haz de luz era quedarse estáticos, lo que facilitó su captura de forma manual. Algunas variantes de este método de muestreo fueron usadas por Negreiros-Fransozo et al. (2002) y Branco et al. (2010).

Los individuos capturados fueron depositados en bolsas plásticas y llevados hasta un lugar resguardado del viento para ser medidos, pesados y diferenciados en tres grupos: jóvenes, hembras y machos. La longitud del carapacho (L, mm) se midió con un Vernier hasta el milímetro más cercano desde la frente hasta el inicio del primer segmento abdominal. El peso húmedo $(\mathrm{P}, \mathrm{g})$ se determinó con una balanza digital con una resolución de 0.01 g. Solo fueron pesados los cangrejos que no habían perdido alguna extremidad durante su captura y manipulación. Para la diferenciación en grupos, se consideraron los siguientes aspectos morfológicos externos: pleópodos ausentes o poco desarrollados (jóvenes); pleópodos desarrollados y telson más ancho que largo (hembras); pleópodos desarrollados y telson más largo que ancho (machos) (Negreiros-Fransozo et al., 2002). Posteriormente, los cangrejos fueron liberados en diferentes lugares de la playa.

La abundancia se determinó como el número de individuos capturados sobre un área de $9000 \mathrm{~m}^{2}$ en cada muestreo. Los meses de muestreo fueron considerados como réplicas para estimar la abundancia. Para la comparación de la abundancia entre playas se realizó un análisis de varianza de una vía seguido de una prueba de Tukey. Se determinó la proporción entre jóvenes y adultos y la proporción entre machos y hembras. Para determinar si la proporción entre machos y hembras es igual $(1: 1)$, se realizó una prueba $\mathrm{X}^{2}$. Las diferencias para todas las pruebas estadísticas fueron consideradas con un nivel de significación de $95 \%$ $(\alpha=0.05)$ (Zar, 1999).

Composición de tallas y relación longitud-peso: La composición de tallas se describió a partir de los datos acumulados de los nueve muestreos en cada playa, elaborando los histogramas de frecuencias de tallas en intervalos de $2 \mathrm{~mm}$. Para estimar el número de grupos de edades relativas en cada histograma, se empleó el método de Bhattacharya (1967) del análisis de progresión modal del paquete FISAT II (Gayanilo, Sparre, \& Pauly, 2005).

Para determinar el crecimiento relativo del cangrejo fantasma en las cuatro playas, se emplearon los datos acumulados de longitud y peso. La relación entre la longitud y el peso se determinó mediante análisis de regresión usando la ecuación potencial $\mathrm{P}=\mathrm{aL}^{\mathrm{b}}$, donde a y b son parámetros de la curva. El parámetro $b$ representa el coeficiente de alometría, indicando que el crecimiento es isométrico cuando $b=3$ o es alométrico negativo o positivo cuando $b<3$ o $b>3$, respectivamente. El grado de asociación entre la longitud y el peso fue calculado por el coeficiente de determinación $\left(r^{2}\right)$. Para comparar el crecimiento relativo de los cangrejos entre las cuatro playas, se realizó un análisis de covarianza (Zar, 1999), donde la variable dependiente es la longitud y el peso es una covariable. Las diferencias significativas se determinaron mediante una prueba de Tukey para datos desbalanceados.

Crecimiento, longevidad y mortalidad: La información básica para la estimación del crecimiento fue obtenida de una serie de histogramas de frecuencias de distribución de tallas. El número de histogramas varió entre las playas debido a que en algunos meses el número de individuos capturados fue muy bajo. En el caso de Majahual no se pudieron determinar los parámetros poblacionales debido al número 
insuficiente de cangrejos recolectados (Sparre \& Venema, 1997). En Aventuras se empleó información de marzo, mayo, agosto y octubre 2013 y de febrero, junio y noviembre 2014. En Xcacel se construyeron histogramas de tallas de marzo, mayo, agosto y octubre 2013 y de febrero, junio, septiembre y noviembre 2014. Los histogramas de frecuencia de tallas de Puerto Ángel se corresponden con los nueve meses de muestreo.

A través del empleo de la rutina ELEFAN I del paquete FISAT II (Gayanilo et al., 2005) se estimaron los parámetros de la ecuación de crecimiento de Von Bertalanffy (ECVB) expresada como: $\mathrm{L}_{\mathrm{t}}=\mathrm{L}_{\infty}\left[1-\mathrm{e}^{(-\mathrm{K}(\mathrm{t}-\mathrm{to})}\right]$; donde $\mathrm{L}_{\mathrm{t}}$ es la talla correspondiente a la edad $t, \mathrm{~L}_{\infty}$ es la longitud máxima teórica, $\mathrm{K}$ es el coeficiente de crecimiento $\mathrm{y}_{\mathrm{o}}$ representa la edad teórica a la longitud cero. La estimación de $t_{o}$ se realizó mediante la ecuación propuesta por Pauly (1979): $\log \left(-\mathrm{t}_{\mathrm{o}}\right)=-0.3922-0.2752\left(\log \mathrm{L}_{\infty}{ }^{-}\right.$ $1.038 \log \mathrm{K})$. La subrutina $K$-scan del ELEFAN I se usó para determinar la ECBV que mejor se ajusta a los datos de distribución de tallas, en este sentido se seleccionó el mayor valor del índice de bondad de ajuste $(R n)$ (Gayanilo et al., 2005). Para comparar los parámetros de crecimiento entre las poblaciones de Aventuras, Xcacel y Puerto Ángel, se calculó el índice de rendimiento en crecimiento $\Phi$ ' (Pauly \& Munro, 1984): $\Phi^{\prime}=2 \log \left(\mathrm{L}_{\infty}\right)+\log (\mathrm{K})$.
El tiempo de vida $\left(\mathrm{t}_{\max }\right)$ teórico para las tres poblaciones de $O$. quadrata fue estimado mediante la inversa de la ecuación de crecimiento de Von Bertalanffy (Taylor, 1958): $\mathrm{t}_{\max }=$ $\left[\operatorname{lnL} \mathrm{L}_{95 \%}-\ln \left(\mathrm{L}_{\infty}-\mathrm{L}_{95 \%}\right)\right] / \mathrm{K}$; donde $\mathrm{L}_{95 \%}$ representa el 95\% de la longitud máxima registrada durante los muestreos. Con los datos acumulados de los muestreos se elaboró la curva de captura en el FISAT II, con el objetivo de estimar la tasa de mortalidad $(Z)$ del modelo de extinción exponencial: $\mathrm{N}_{\mathrm{t}}=\mathrm{N}_{\mathrm{t}}-1(\mathrm{e}-\mathrm{Z} \Delta \mathrm{t})$; donde $\mathrm{N}_{\mathrm{t}}$ es el número de individuos en el tiempo $\mathrm{t}$ y $\mathrm{Z}$ es el parámetro de curvatura de la ecuación.

\section{RESULTADOS}

Variables abióticas: Las cuatro playas clasifican como reflectivas con valores de $\Omega=0.6-1.1$, con un tamaño mediano del sedimento $\mathrm{Md}_{50}=0.32-0.38 \mathrm{~mm}$. La pendiente en Majahual y Puerto Ángel es moderada, con valores significativamente menores que en Aventuras y Xcacel. La mayor amplitud de la resaca es en Xcacel y Puerto Ángel. En esta última, la cantidad de materia orgánica en el sedimento fue significativamente mayor que en Aventuras y Majahual (Cuadro 1).

Abundancia: Se recolectó un total de 1047 cangrejos durante nueve meses de muestreo. En cada una de las cuatro playas se recolectaron

CUADRO 1

Variables abióticas (media $\pm \mathrm{DE}$ ) de las cuatro playas estudiadas en la costa del Caribe mexicano

TABLE 1

Abiotic variables (mean $\pm \mathrm{SD}$ ) of the four studied sandy beaches from the coast of the Mexican Caribbean

\begin{tabular}{lcccc}
\multicolumn{1}{c}{ Características } & Aventuras & Xcacel & Majahual & Puerto Angel \\
Ancho $(\mathrm{m})$ & $20.9 \pm 4.9(\mathrm{a})$ & $30.4 \pm 8.7$ (b) & $29.8 \pm 4.4(\mathrm{~b})$ & $17.5 \pm 3.4(\mathrm{a})$ \\
Pendiente $\left({ }^{\circ}\right)$ & $7.9 \pm 2.2(\mathrm{a})$ & $7.2 \pm 2.4(\mathrm{a})$ & $3.7 \pm 1.4(\mathrm{~b})$ & $3.6 \pm 1.5(\mathrm{~b})$ \\
Amplitud de resaca $(\mathrm{m})$ & $3.7 \pm 2.3(\mathrm{a})$ & $5.5 \pm 1.4(\mathrm{~b})$ & $0.7 \pm 0.6(\mathrm{c})$ & $5.4 \pm 2.5(\mathrm{~b})$ \\
$\mathrm{Md}_{50}(\mathrm{~mm})$ & 0.38 & 0.34 & 0.38 & 0.32 \\
$\Omega$ & 1.1 & 0.8 & 0.6 & 0.8 \\
MOT $(\%)$ & $1.6 \pm 0.3(\mathrm{a})$ & $1.9 \pm 1.5(\mathrm{ab})$ & $1.6 \pm 0.3(\mathrm{a})$ & $2.3 \pm 0.5(\mathrm{~b})$ \\
\hline
\end{tabular}

$\mathrm{Md}_{50}$ : tamaño del sedimento; $\Omega$ : parámetro de Dean; MOT: materia orgánica total.

$\mathrm{Md}_{50}$ : grain size; $\Omega$ : Dean's parameter; MOT: total organic matter.

Las letras diferentes en cada fila representan diferencias significativas (Tukey, $\mathrm{p}<0.05$ ).

Different letters in the same row indicate significant differences (Tukey, $\mathrm{p}<0.05$ ). 
más individuos durante el primer año de muestreo que durante el segundo año. La mayor abundancia se encontró en Puerto Ángel y la menor en Majahual, existiendo diferencias significativas entre estas dos playas (Tukey, $\mathrm{p}<0.05$ ) (Cuadro 2). Los resultados de la prueba de Tukey evidenciaron que, aunque la abundancia en Aventuras fue menor que en Xcacel, no hubo diferencia significativa entre ambas playas; sin embargo, la diferencia entre estas y las otras playas fue marginal $(0.05<\mathrm{p}<0.10)$.

La proporción de sexos fue de 1:1 en Puerto Ángel, Majahual y Xcacel $\left(X^{2}, p>0.05\right)$, mientras que en Aventuras fue de 0.7 machos por cada hembra $\left(\mathrm{X}^{2}, \mathrm{p}<0.05\right)$. La proporción entre jóvenes y adultos fue de 1:1 en Xcacel y Aventuras. En Majahual se recolectaron dos jóvenes por cada adulto, y en Puerto Ángel esta proporción resultó ser muy diferente, con 0.2 jóvenes por cada adulto.

Composición de tallas y relación longitud-peso: En Xcacel la estructura de tallas osciló entre 4-32 $\mathrm{mm}$; en el resto de las playas la talla mínima encontrada fue de $5 \mathrm{~mm}$.
La talla máxima para Aventuras, Majuahual y Puerto Ángel fue de $27 \mathrm{~mm}, 25 \mathrm{~mm}$ y $30 \mathrm{~mm}$, respectivamente. La descomposición de grupos modales por el método de Batthacharya para los datos agrupados de longitud, reveló la presencia de al menos dos grupos de edades en todas las playas (Fig. 2). En Xcacel, el primer grupo presentó una longitud media ( \pm desviación estándar) de $10.6 \pm 2.3 \mathrm{~mm}$, y el segundo una longitud media de 20.1 $\pm 2.8 \mathrm{~mm}$. En Aventuras, el primer grupo presentó una talla media de $7.0 \pm 1.1 \mathrm{~mm}$ y el segundo de $18.0 \pm 2.9 \mathrm{~mm}$. En Majahual, el primer grupo se detectó alrededor de $9.8 \pm 2.6 \mathrm{~mm}$ y el segundo en $20.0 \pm 2.0 \mathrm{~mm}$. En Puerto Angel, el histograma de frecuencias con los datos acumulados mostró un solo grupo de edades con un pico alrededor de los $20 \mathrm{~mm}$; sin embargo, la descomposición modal arrojó como resultado la presencia de tres grupos de tallas: $12.5 \pm 2.6 \mathrm{~mm}, 19.8 \pm 2.0 \mathrm{~mm}$ y $25.0 \pm 2.5$ mm. En Xcacel, Aventuras y Majahual pudiera existir un tercer grupo de tallas pero en la descomposición modal este no se estimó debido al bajo número de individuos.

CUADRO 2

Estadística descriptiva de la abundancia de Ocypode quadrata en las cuatro playas estudiadas en la costa del Caribe mexicano

TABLE 2

Descriptive statistics of Ocypode quadrata abundance for the four studied sandy beaches from the coast of the Mexican Caribbean

\begin{tabular}{lcccc}
\multicolumn{1}{c}{ Mes } & Xcacel & Puerto Angel & Aventuras & Majahual \\
Marzo/2013 & 42 & 43 & 51 & 31 \\
Mayo/2013 & 41 & 60 & 20 & 23 \\
Agosto/2013 & 41 & 73 & 32 & 22 \\
Octubre/2013 & 42 & 55 & 33 & 9 \\
Febrero/2014 & 27 & 15 & 17 & 5 \\
Abril/2014 & 1 & 45 & 7 & 9 \\
Junio/2014 & 36 & 51 & 38 & 0 \\
Septiembre/2014 & 23 & 46 & 12 & 6 \\
Noviembre/2014 & 18 & 413 & 40 & 113 \\
Total & 271 & $45.9(\mathrm{a})$ & 250 & $12.6(\mathrm{~b})$ \\
Media & $30.1(\mathrm{ab})$ & 17.5 & $27.8(\mathrm{ab})$ & 10.3 \\
Desviación estándar & 14.2 & & 14.6 & 8 \\
\hline
\end{tabular}

Letras diferentes (entre paréntesis) representan diferencias significativas (Tukey, $\mathrm{p}<0.05$ ).

Different letters (in brackets) indicate significant differences (Tukey, $\mathrm{p}<0.05$ ). 


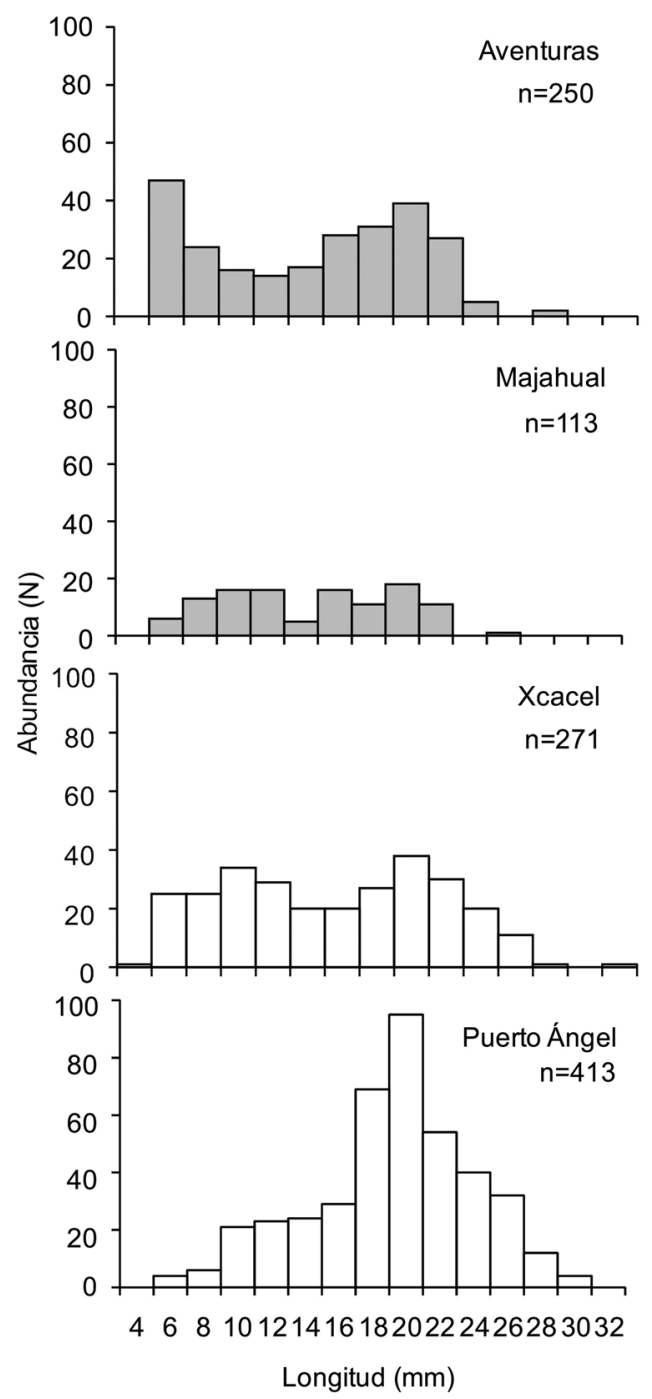

Fig. 2. Ocypode quadrata. Frecuencia de tallas (datos agrupados) en cuatro playas del Caribe mexicano. Las barras grises representan a las playas con mayor disturbio humano y las blancas a las más conservadas.

Fig. 2. Ocypode quadrata. Length frequency (pooled data) at four beaches of the Mexican Caribbean. Grey bars represent highly disturbed beaches, and white bars represent beaches with higher conservation values.

En las cuatro playas se recolectaron individuos con tallas menores de $10 \mathrm{~mm}$ en casi todos los meses de muestreo; excepto en Xcacel y Aventuras, durante abril 2013 y en Puerto Ángel, durante mayo y agosto 2013. Este resultado indica que el reclutamiento de esta especie, en las playas estudiadas, es continuo durante todo el año. Sin embargo, solo encontramos hembras con masas de huevos en Aventuras (una en octubre de 2013 y otra en abril 2014) y en Puerto Ángel, durante agosto 2013 (cuatro), octubre 2013 (dos), abril 2014 (dos) y noviembre 2014 (dos). Sólo tres cópulas fueron observadas (en Puerto Ángel durante agosto 2013), y estas coincidieron con luna llena.

La relación entre la longitud y el peso de los cangrejos fue altamente significativa para las poblaciones de las cuatro playas (Cuadro 3, Fig. 3). El coeficiente de alometría (b) reveló que en Xcacel y Puerto Ángel el crecimiento es isométrico, mientras en Aventuras y Majahual es alométrico negativo. Los cangrejos alcanzan mayor talla y peso en las dos playas mejor conservadas (Fig. 3). Mediante el ANCOVA se determinó que existen diferencias en la relación entre la talla y el peso entre la población de cangrejos de Puerto Ángel con respecto al resto de las playas (Tukey, $\mathrm{p}<0.05$ ), así como entre Xcacel y Majahual (Cuadro 3).

\section{Crecimiento, longevidad y mortalidad:} Debido al bajo número de individuos recolectados en Majahual, se decidió realizar la comparación de los parámetros poblacionales entre el resto de las playas. Los valores de $\mathrm{K}$ oscilaron entre 0.6-0.97/año y la $\mathrm{L}_{\infty}$ entre 29.0-33.6 mm. Los valores de $t_{0}$ variaron entre $0.16-0.27 / a n ̃ o$ (Cuadro 4). Con estos datos se construyeron las curvas de crecimiento de $O$. quadrata para las tres playas (Fig. 4). En Xcacel el crecimiento resultó ser mayor, alcanzando tallas de $15 \mathrm{~mm}$ durante el primer año de vida; mientras en Puerto Ángel y Aventuras el crecimiento de los cangrejos fue más lento (Cuadro 4, Fig. 4).

Los mayores valores de mortalidad se encontraron en Xcacel y Aventuras (Cuadro 4). Adicionalmente, el tiempo de vida estimado fue de 2.3, 3.2 y 3.4 años para Xcacel, Puerto Ángel y Aventuras, respectivamente.

\section{DISCUSIÓN}

La mayor parte de los estudios para estimar la abundancia de $O$. quadrata, se han basado 


\section{CUADRO 3}

Parámetros (media \pm EE) de la relación longitud-peso para las poblaciones de Ocypode quadrata en cuatro playas del Caribe mexicano

TABLE 3

Parameters (mean $\pm \mathrm{SE}$ ) of the length-weight relationship of Ocypode quadrata for four beaches of the Mexican Caribbean populations

\begin{tabular}{ccccc} 
Parámetros & Xcacel & Puerto Ángel & Aventuras & Majahual \\
$a$ & $-6.79(0.084)$ & $-7.04(0.133)$ & $-6.49(0.132)$ & $-6.13(0.147)$ \\
$b$ & $2.98(0.029)$ & $3.06(0.050)$ & $2.85(0.05)$ & $2.73(0.054)$ \\
$\mathrm{R}^{2}$ & 0.97 & 0.94 & 0.95 & 0.97 \\
ANCOVA & & \multicolumn{2}{c}{$\mathrm{F}=7.08 ; \mathrm{p}=0.0001$} & (c) \\
Prueba a posteriori & (a) & (b) & & (ac)
\end{tabular}

Las letras diferentes (entre paréntesis) representan diferencias significativas (Tukey para datos desbalanceados, $\mathrm{p}<0.05$ ) Different letters (in brackets) indicate significant differences (Tukey for unequal $\mathrm{N}, \mathrm{p}<0.05$ ).

En todos los casos las regresiones y los parámetros estimados fueron estadísticamente significativos $(\mathrm{p}<0.05)$. Se muestran los resultados del análisis de covarianza (ANCOVA, con datos transformados) de la relación longitud peso, y de la prueba a posteriori.

Regressions and parameter estimates were significant in all cases $(\mathrm{p}<0.05)$. The analysis of cavariance results (ANCOVA, with transformed data) of the length-weight relationship, and the post hoc test, are also shown.

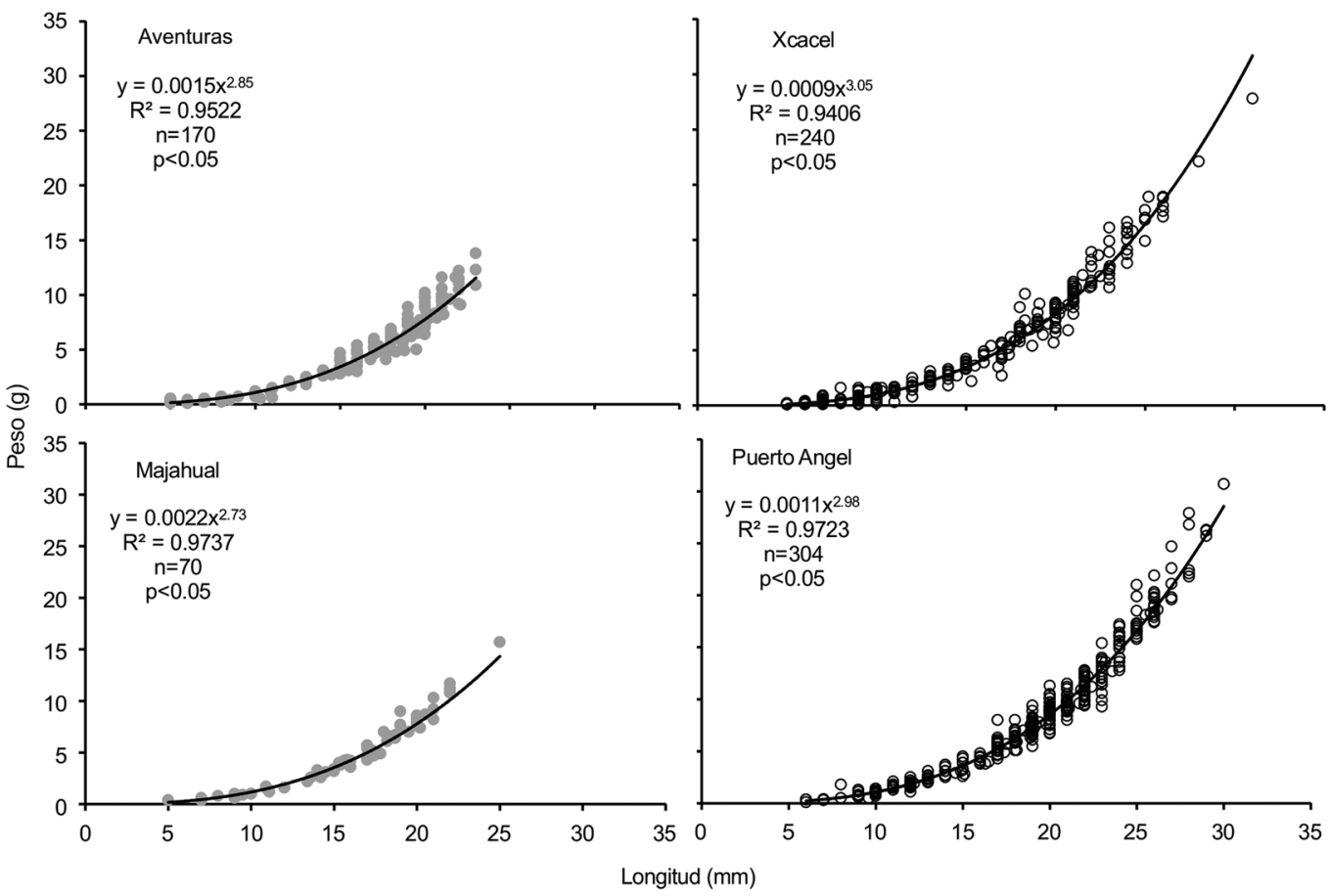

Fig. 3. Ocypode quadrata. Modelos ajustados de la relación longitud-peso en cuatro playas del Caribe mexicano. Los puntos grises representan a las playas con mayor disturbio humano y los círculos blancos representan a las playas más conservadas. Fig. 3. Ocypode quadrata. Fitted models of the length-weight relationship at four beaches of the Mexican Caribbean. Grey dots represent highly disturbed beaches, and white circles represent beaches with higher conservation values. 


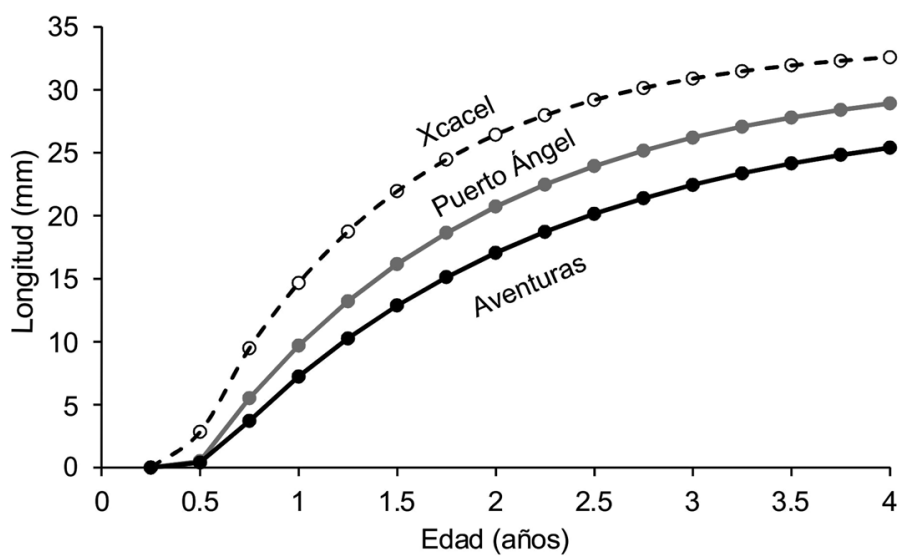

Fig. 4. Ocypode quadrata. Curvas de crecimiento ajustadas al modelo de crecimiento de Von Bertalanffy en tres playas del Caribe mexicano. Los parámetros del modelo se muestran en el Cuadro 4.

Fig. 4. Ocypode quadrata. Growth curves fitted to the Von Bertalanffy growth model in three beaches of the Mexican Caribbean. Model parameters are shown in Table 4.

CUADRO 4

Ocypode quadrata. Parámetros poblacionales para tres playas del Caribe mexicano

TABLE 4

Ocypode quadrata. Population parameters for three beaches of the Mexican Caribbean

\begin{tabular}{lccccccccc}
\multicolumn{1}{c}{ Playa } & $\mathrm{L}_{\max }$ & $\mathrm{L}_{95 \%}$ & $\mathrm{~L}_{\infty}$ & $\mathrm{K}$ & $R n$ & $\Phi^{\prime}$ & $\mathrm{t}_{\mathrm{o}}$ & $\mathrm{Z}$ & 2.81 \\
Aventuras & 27 & 25.6 & 29.0 & 0.60 & 0.32 & 2.70 & 0.27 & 2.94 \\
Xcacel & 32 & 30.4 & 33.6 & 0.97 & 0.36 & 3.04 & 0.16 & 2.94 \\
Puerto Angel & 30 & 28.5 & 31.6 & 0.70 & 0.34 & 2.84 & 0.23 & 1.85 \\
\hline
\end{tabular}

$\mathrm{L}_{\max }$ : longitud máxima encontrada $(\mathrm{mm}) ; \mathrm{L}_{95 \%}: 95 \%$ de $\mathrm{L}_{\max } ; \mathrm{L}_{\infty}$ : longitud máxima teórica $(\mathrm{mm}) ; \mathrm{K}$ : parámetro de la curva (1/año); Rn índice de bondad de ajuste; $\Phi$ ': índice de rendimiento en crecimiento; $\mathrm{t}_{\mathrm{o}}$ : edad teórica a la longitud cero (1/año); Z: tasa de mortalidad (1/año).

$\mathrm{L}_{\max }$ : maximun carapace length; $\mathrm{L}_{95 \%}: 95 \%$ of $\mathrm{L}_{\max } ; \mathrm{L}_{\infty}$ : asymptotic length $(\mathrm{mm}) ; \mathrm{K}$ : curvature parameter (1/year); Rn: goodness of fit index; $\Phi^{\prime}$ : standard growth index; $t_{0}$ : age at cero length (1/year); Z: mortality rate (1/year).

en el censo de galerías y no en el conteo de individuos. Solo tres trabajos han reportado la abundancia de cangrejos basado en el conteo de los organismos (Steiner \& Leatherman, 1981; Negreiros-Fransozo et al., 2002; Branco et al., 2010). Debido a que el esfuerzo de muestreo fue diferente en estos trabajos mencionados, no es posible comparar la abundancia de cangrejos en playas de Quintana Roo con reportes de la literatura. Sin embargo, en este estudio se encontró que la mayor abundancia de cangrejos se presenta en las playas más conservadas y expuestas, similar a lo reportado por Steiner y Leatherman (1981). Este hallazgo también concuerda con los estudios basados en el conteo indirecto, donde se reporta que las playas más perturbadas por actividades humanas presentan menor abundancia de galerías (Blankensteyn, 2006; Neves \& Bemvenuti, 2006; Araújo et al. 2008; Souza, Lavoie, Bonifácio, \& Rocha, 2008; Magalhães, Lima, Barros, \& Dominguez, 2009).

Una de las razones por la cual no hay diferencias en la abundancia entre Aventuras y Xcacel podría ser que, en la primera aún existen remanentes de dunas. Se ha demostrado que el estado de las dunas es un factor importante en la conservación de los cangrejos fantasma 
(Barros, 2001; Noriega, Schlacher, \& Smeuninx, 2012). La destrucción de la duna, las actividades de limpieza de playa, así como la construcción de infraestructuras para el turismo en Majahual, deben ser las principales causas del bajo número de cangrejos en este sitio, debido a la limitación del espacio disponible para construir galerías.

Por otra parte, varios factores conllevan a un alto número de cangrejos en Puerto Ángel; en esta playa casi no existe influencia humana y además hay alta disponibilidad de alimento, reflejado en una mayor cantidad de materia orgánica en el sedimento. Souza et al. (2008) encontraron que el contenido de materia orgánica en el sedimento se relaciona positivamente con la abundancia de cangrejos. Otra posible explicación pudiera ser que Puerto Ángel y Xcacel, son playas más expuestas al oleaje y con mayor dinámica, lo que puede favorecer el arribo de nuevos reclutas a estas playas. En playas expuestas se ha descrito que algunas fuerzas hidrodinámicas como las corrientes de retorno y los giros, favorecen el transporte larval y, por tanto, el arribo de nuevos reclutas (Caddy \& Defeo, 2003).

La proporción sexual siguió la relación esperada entre machos y hembras, no obstante en Aventuras el número de hembras fue mayor. Negreiros-Fransozo et al. (2002) reportaron una proporción 1:1 en una playa con poco disturbio humano. Otros trabajos en playas de Brasil describen que la proporción equilibrada entre hembras y machos puede ocurrir en algunos meses, pero de forma general se han encontrado más machos que hembras (Alberto \& Fontoura, 1999; Branco et al., 2010; Silva \& Calado, 2013; Corrêa et al., 2014). En una playa de Veracruz, México, se reportó también que la proporción de machos era mayor (Valero-Pacheco et al., 2007). En la literatura no se han discutido las posibles causas de una proporción desbalanceada entre hembras y machos de $O$. quadrata, no obstante los trabajos mencionados anteriomente fueron realizados en playas con muy poca influencia humana. Trott (1998) investigó la proporción de sexos de $O$. gaudichaudii en cuatro playas del Pacífico de Costa Rica y encontró que machos y hembras pueden seguir un patrón de comportamiento diferente. Las hembras de cangrejos fantasma pueden permanecer más tiempo que los machos dentro de las galerías; este comportamiento ocurre durante la fase de desarrollo de los huevos (Trott, 1998; Negreiros-Fransozo et al., 2002), lo que pudiera explicar el mayor número de machos capturados en otros estudios. Sin embargo, en Aventuras se encontró un patrón inverso, lo que sugiere que en esta playa los machos sufren mayor mortalidad que las hembras. Teniendo en cuenta que los machos son más activos en la playa, el menor número encontrado puede ser atribuido a dos causas: 1) la influencia de los predadores como mapaches (Procyon lotor) y aves de la familia Ardeidae, cuyas huellas fueron encontradas en la playa; 2) la limpieza mecánica de la playa que puede ocasionar la muerte por aplastamiento. Teniendo en cuenta que las huellas de predadores fueron encontradas en las otras tres playas también, el mayor número de hembras encontrados en Aventuras podría ser un indicador del efecto de la limpieza mecanizada en esta playa. Se ha demostrado que el uso de vehículos motorizados pueden ser responsables de una alta mortalidad por aplastamiento de los cangrejos en las playas (Schlacher, Thompson, \& Price, 2007).

Fisher y Tevesz (1981) atribuyeron que la desproporción entre jóvenes y adultos de cangrejo fantasma, puede estar relacionada con el nivel de impacto. Sin embargo, sus resultados fueron cuestionados por Leber (1981), debido a que la categorización de playas de acuerdo al nivel de disturbio, y los análisis estadísticos correspondientes, no eran correctos. En playas conservadas de Brasil se encontró que la proporción entre jóvenes y adultos era 1:1 (Alberto \& Fontoura, 1999) o que el número de adultos era más de dos veces mayor (Negreiros-Fransozo et al., 2002; Branco et al., 2010; Corrêa et al., 2014), sugiriendo una mayor sobrevivencia de cangrejos en estas playas. La proporción entre jóvenes y adultos se corresponde con las tasas de mortalidad estimadas para Aventuras, Xcacel y Puerto Ángel; por tanto, el menor 
número de adultos encontrados en Majahual con respecto al número de jóvenes, podría atribuirse a una mayor mortalidad de cangrejos en esta playa. En Majahual, la arena está muy compactada debido al pisoteo y a la limpieza con rastrillos; por otra parte, durante los muestreos se constató que las galerías de los cangrejos son poco profundas, extendiéndose de forma casi horizontal al substrato. Teniendo en cuenta que los cangrejos que viven en galerías poco profundas son más subceptibles a sufrir daños (Schlacher et al., 2007), el nivel de disturbio en Majahual podría explicar por qué pocos jóvenes llegan a convertirse en adultos en esta playa. Un escenario diferente se presenta en Puerto Ángel, donde casi no existe perturbación antrópica, y hay mayor disponibilidad de alimento; en esta playa los cangrejos crecen rápido y tienen mayor sobrevivencia resultando en una mayor proporción de adultos.

También se encontró que el crecimiento de los cangrejos pudiera estar relacionado con la remoción de la materia orgánica producto de la limpieza de playas. La condición corporal de los cangrejos mostró marcadas diferencias (resultados del ANCOVA) entre Puerto Ángel y el resto de las playas, sugiriendo que el contenido de materia orgánica en el sedimento se relaciona directamente con el crecimiento de estos organismos. La talla y el peso de los cangrejos aumentó desde la playa más perturbada (Majahual) hasta la playa más conservada y con mayor disponibilidad de alimento (Puerto Ángel). En Aventuras y Majahual los cangrejos manifestaron un crecimiento mayor en talla que en peso, indicando que en estas playas son menos robustos, en comparación con Xcacel y Puerto Ángel. A pesar que la cantidad de materia orgánica no presenta diferencias significativas entre Xcacel, Aventuras y Majahual, sí se observó que en la primera existe mayor disponibilidad de alimento debido a la alta acumulación de macrófitos en la línea de deriva. Además, en otro estudio (Schlacher, de Jargen, \& Nielsen, 2011) se reportó que la talla y la condición corporal de los cangrejos fantasma está relacionada con la cantidad de alimento disponible. Por otra parte, la reducción del tamaño de las galerías, usado como indicador del tamaño de los individuos, es una respuesta típica de los cangrejos fantasma ante el incremento del disturbio antrópico (Lucrezi \& Schlacher, 2014).

De acuerdo a los valores de $\Phi$ ', el crecimiento de los cangrejos de Xcacel es el mayor, seguido de las poblaciones de Puerto Ángel y Aventuras. La tasa de crecimiento de $O$. quadrata solo se ha reportado bajo condiciones de laboratorio (Haley, 1972). Por otra parte, un solo estudio (Pombo, 2015) ha estimado parámetros de crecimiento en poblaciones naturales de O. quadrata; en el cual se reportaron valores de K (0.87-1.11) y del índice de rendimiento en crecimiento $\left(\Phi^{\prime}=3.29-3.49\right)$ mayores que los encontrados en el presente trabajo. Sin embargo, la autora reconoce que esos valores pueden haber sido sobre-estimados debido y que los datos se basaron en el tamaño de las galerías y no de los individuos.

Las diferencias en la tasa de crecimiento entre Xcacel y Puerto Ángel puede estar asociada a procesos dependientes de la densidad. En Xcacel se observó una disponibilidad de alimento menor que en Puerto Ángel, pero la abundancia de cangrejos en esta última playa fue mayor, lo que implica mayor competencia por el alimento y en consecuencia menor crecimiento. Sin embargo, la limpieza de playa Aventuras disminuye la disponibilidad de alimento lo que podría resultar en un menor crecimiento de los cangrejos. La importancia relativa de diferentes factores sobre los parámetros poblacionales de especies de playas arenosas ya fue discutida por Defeo y Maclachlan (2005). Estos autores analizaron diferentes estudios sobre dinámica poblacional de especies de invertebrados y propusieron que en playas donde no existen disturbios, las poblaciones están controladas por procesos dependientes de la densidad, siendo las relaciones bióticas muy importantes en regular el crecimiento y la mortalidad. Sin embargo, en playas perturbadas, el ambiente físico controla los parámetros poblacionales.

La mayor mortalidad fue estimada para la población de Xcacel, aunque con poca 
diferencia de la mortalidad estimada en Aventuras. Teniendo en cuenta que Xcacel está bastante bien conservada, atribuimos la alta mortalidad a causas naturales como la influencia de los predadores. Branco et al. (2010) sugirieron que en playas con poco impacto humano, la presión ejercida por los predadores puede contribuir substancialmente a la disminución de la población de cangrejos. Por otra parte, el tiempo de vida estimado para los cangrejos de Aventuras, Xcacel y Puerto Ángel, coincide con el análisis de descomposición modal de los histogramas de frecuencia de tallas. Estos estimados coinciden también con la longevidad de tres años reportada para esta especie en ambos extremos de su ámbito de distribución (Haley, 1972; Alberto \& Fontoura, 1999).

El nivel de disturbio humano puede tener una influencia importante en regular no solo la abundancia de $O$. quadrata, sino también algunas características de la historia de vida de esta especie. Otros factores como las relaciones inter e intraespecíficas pueden tener menor importancia en regular los parámetros poblacionales del cangrejo fantasma en playas impactadas. Además puede existir un efecto conjunto de las características físicas intrínsecas de cada playa con el disturbio humano, en controlar la historia de vida de las poblaciones de esta especie. Una compilación reciente (Petracco, Cardoso, \& Turra, 2013) demostró que a nivel global las características morfodinámicas de las playas no presentan relación alguna con descriptores poblacionales como la masa corporal, la longevidad y la tasa de renovación (cociente entre la producción secundaria y la biomasa). Quizás en estudios a grandes escalas, las características morfodinámicas no sean relevantes sobre la dinámica poblacional. En cambio, entre playas dentro de una misma región pudieran existir algunas limitaciones metodológicas que no permiten separar la contribución relativa de los diferentes factores sobre la dinámica poblacional de $O$. quadrata. En este sentido, en el presente trabajo, las dos playas impactadas por el turismo presentan cierto grado de protección ofrecido por el rompeolas en Xcacel y por la cresta arrecifal y el muelle en Majahual. Estas barreras hacen que ambas playas presenten una dinámica diferente de las otras dos (Xcacel y Puerto Ángel) y pudieran estar limitando el arribo de larvas a las playas, lo que puede influir sobre la abundancia de esta especie.

Las playas estudiadas pueden ser categorizadas como reflectivas de acuerdo al valor de $\Omega$. Sin embargo, este índice fue propuesto para playas oceánicas expuestas dominadas por el oleaje (Short, 1996) y no se ajusta a las características reales de las playas del Caribe mexicano. El marco teórico sobre la ecología en playas protegidas de baja energía aún está en desarrollo (Torres, 2010) y se requieren diseños más robustos para superar las limitaciones metodológicas que tiene el presente trabajo. Por otra parte, en este estudio no se pudo discernir entre los efectos del pisoteo, la limpieza de la playa y la afectación a las dunas por lo que se asume que la suma de estas acciones afectan la dinámica poblacional del cangrejo fantasma. Es necesario resaltar que el análisis realizado conduce a considerar que la eliminación de la energía de subsidio de estas playas, debido a la limpieza constante, es un factor importante en regular el crecimiento de esta especie.

Los resultados de este trabajo tienen relevancia desde el punto de vista del manejo de las playas arenosas. Varios estudios que han comparado la abundancia de $O$. quadrata entre playas perturbadas y conservadas, han arribado a la conclusión de que las actividades humanas conllevan a la disminución de la abundancia de esta especie (Steiner \& Leatherman, 1981; Turra, Gonçalves, \& Denadai, 2005; Blankensteyn, 2006; Neves \& Bemvenuti, 2006; Araújo et al., 2008; Hobbs, Landry, \& Perry, 2008; Souza et al., 2008). El presente estudio ha demostrado que el disturbio humano puede impactar negativamente otros atributos de las poblaciones como el crecimiento y la sobrevivencia de los individuos. Aunque las condiciones físicas de las playas pueden obscurecer los efectos del impacto humano, se considera que el cangrejo fantasma es un buen indicador del disturbio en playas arenosas, ya que sus respuestas poblacionales pueden ser medibles 
y comparables. En este sentido, se propone incluir otras variables poblacionales como la condición corporal, el crecimiento y la mortalidad, en estudios que involucren el uso de $O$. quadrata como indicador de la calidad y/o estado de las playas arenosas. Además, de tener en cuenta la realización de diseños más robustos que consideren a las características físicas de las playas, como una variable relevante en la regulación de la abundancia del cangrejo fantasma.

Por otra parte, se recomienda la adopción de medidas para controlar la ejecución de actividades de limpieza en las playas turísticas. Se debe tomar conciencia que el impacto mecánico durante la limpieza de playas puede infligir altas mortalidades a los organismos de forma directa; y de manera indirecta remueve una energía de subsidio que es usada como soporte vital para los organismos. Además, estos impactos pueden desencadenar mecanismos de cascada, involucrando a otros animales dentro de la cadena trófica en las playas arenosas.

\section{AGRADECIMIENTOS}

Agradecemos la colaboración de Roberto Herrera durante el trabajo de campo, así como al personal de la Reserva Estatal Xcacel por permitirnos el acceso y la investigación en el área. Este trabajo forma parte de la tesis de doctorado de Frank A. Ocaña quien cuenta con una beca del Consejo Nacional de Ciencia y Tecnología de México (CONACYT).

\section{RESUMEN}

El cangrejo fantasma Ocypode quadrata, tiene una alta relevancia ecológica en las playas del Atlántico occidental y se ha propuesto como un indicador del impacto humano sobre este ecosistema. Sin embargo, no se han evaluado los efectos del disturbio humano sobre su dinámica poblacional. En este trabajo se compara la abundancia, el crecimiento y la mortalidad de $O$. quadrata en dos playas perturbadas (Aventuras y Majahual) y dos playas conservadas (Xcacel y Puerto Ángel) de las costas del Caribe mexicano. Se realizaron nueve muestreos en cada playa (marzo/2013-noviembre/2014). Durante las primeras horas de la noche se recolectaron cangrejos, de forma manual por tres personas, en un área aproximada de $9000 \mathrm{~m}^{2}$.
Los cangrejos fueron medidos, pesados y separados en tres grupos: jóvenes, hembras y machos. La mayor abundancia de cangrejos se encontró en las playas conservadas pero solo se observaron diferencias significativas entre Puerto Ángel y Majahual (Tukey, $\mathrm{p}<0.05$ ). La proporción jóvenesadultos fue 1:1 en Xcacel y Aventuras. En Majahual se recolectó un mayor número de jóvenes que adultos y en Puerto Ángel el número de adultos triplicó el de jóvenes. La talla máxima para Aventuras, Xcacel, Majahual y Puerto Ángel fue de 27, 32, 25 y $30 \mathrm{~mm}$, respectivamente. En las playas se encontraron entre dos y tres grupos de edades diferentes. En las cuatro playas se recolectaron individuos con tallas menores de $10 \mathrm{~mm}$ en casi todos los meses de muestreo, indicando que el reclutamiento es continuo durante todo el año. La relación entre la longitud y el peso del cangrejo fantasma fue isométrica en las playas mejor conservadas y alométrica negativa en las playas impactadas. Los cangrejos de Puerto Ángel mostraron mejor condición corporal que en el resto de las playas (Tukey, $\mathrm{p}<0.05$ ). Los valores de $\mathrm{K}$ oscilaron entre 0.6-0.97/año y la $\mathrm{L}_{\infty}$ entre 29.0-33.6 mm. Los cangrejos en las dos playas conservadas mostraron mayores parámetros de crecimiento en comparación con las dos playas perturbadas. Los mayores valores de mortalidad se encontraron en Xcacel y Aventuras. En las playas perturbadas, la destrucción de las dunas, la limpieza mecánica y la remoción de materia orgánica, parecen ser las principales causas que conllevan a una baja abundancia de cangrejos, un menor crecimiento y una alta mortalidad. Sin embargo en las playas mejor conservadas, las relaciones biológicas parecen ser más importantes en regular la dinámica poblacional de esta especie. Estos resultados tienen relevancia desde el punto de vista de manejo de las playas arenosas, ya que el cangrejo fantasma es un buen indicador del disturbio humano y sus respuestas poblacionales son medibles y comparables.

Palabras clave: cangrejo fantasma, playas arenosas, indicador biológico, crecimiento, mortalidad.

\section{REFERENCIAS}

Alberto, R. M. F., \& Fontoura, N. F. (1999). Distribuição e estrutura etária de Ocypode quadrata (Fabricius, 1787) (Crustacea, Decapoda, Ocypodidae) em praia arenosa do litoral sul do Brasil. Revista Brasileira de Biologia, 59, 95-108.

Antunes, G. F., do Amaral, A. P. N., Ribarcki, F. P., de Fátima-Willand, E., Zancan, D. M., \& Vinagre, A. S. (2010). Seasonal variations in the biochemical composition and reproductive cycle of the ghost crab Ocypode quadrata (Fabricius 1787) in southern Brazil. Journal of Experimental Zoology A: Ecological Genetics and Physiology, 313, 280-291.

Araújo, C. C. V., Rosal, D. M., \& Fernandes, J. M. (2008). Densidade e distribuição espacial do caranguejo Ocypode quadrata (Fabricius, 1787) (Crustacea, 
Ocypodidae) em três praias arenosas do Espírito Santo, Brasil. Biotemas, 21, 73-80.

Barros, F. (2001). Ghost crabs as a tool for rapid assessment of human impacts on exposed sandy beaches. Biological Conservation, 97, 399-404.

Barton, B. T., \& Roth, J. D. (2008). Implications of intraguild predation for sea turtle nest protection. Biological Conservation, 141, 2139-2145.

Bhattacharya, C. G. (1967). A simple method of resolution of a distribution into Gaussian components. Biometrics, 23, 115-135.

Blankensteyn, A. (2006). O uso do caranguejo mariafarinha Ocypode quadrata (Fabricius) (Crustacea, Ocypodidae) como indicador de impactos antropogênicos em praias arenosas da Ilha de Santa Catarina. Revista Brasileira de Zoologia, 23, 870-876.

Branco, J. O., Hillesheim, J. C., Fracasso, H. A. A., Christoffersen, M. L., \& Evangelista, C. L. (2010). Bioecology of the ghost crab Ocypode quadrata (Fabricius, 1787) (Crustacea: Brachyura) compared with other intertidal crabs in the southwestern Atlantic. Journal of Shellfish Research, 29, 503-512.

Buchanan, J. B. (1984). Sediment analysis. In N. S. Holme, \& A. D. McIntyre (Eds.), Methods for the Study of Marine Benthos (pp. 41-65). Boston, USA: Blackwell Scientific.

Caddy, J. F., \& Defeo, O. (2003). Enhancing or restoring the productivity of natural populations of shellfish and other marine invertebrate resources. Rome, Italy: FAO Fish Technical Papers 448.

Carrillo, L., Palacios-Hernández, E., Yescas, M., \& Ramírez-Manguilar, A. M. (2009). Spatial and Seasonal Patterns of Salinity in a Large and Shallow Tropical Estuary of the Western Caribbean. Estuaries and Coasts, 32, 906-916.

Corrêa, M. O. D. A., Andrade, L. S., Costa, R. C., Castilho, A. L., Bertini, G., \& Fransozo, A. (2014). Vertical distribution by demographic groups of ghost crab Ocypode quadrata (Crustacea: Brachyura). Biologia Section Zoology, 69, 905-915.

Defeo, O., \& McLachlan, A. (2005). Patterns, processes and regulatory mechanisms in sandy beach macrofauna: a multi-scale analysis. Marine Ecology Progress Series, 295, 1-20.

Defeo, O., \& McLachlan, A. (2011). Coupling between macrofauna community structure and beach type: a deconstructive meta-analysis. Marine Ecology Progress Series, 433, 29-41.

Díaz, H., \& Costlow, J. D. (1972). Larval development of Ocypode quadrata (Brachyura: Crustacea) under laboratory conditions. Marine Biology, 15, 120-131.
Fisher, J. B., \& Tevesz, M. J. S. (1979). Within-habitat spatial patterns of Ocypode quadrata (Fabricius) (Decapoda, Brachyura). Crustaceana, 5S, 31-36.

Gayanilo, F. C., Sparre, P., \& Pauly, D. (2005). FAOICLARM Stock Assessment Tools II (FiSAT II). User's guide. Rome, Italy: FAO Computational Information Series for Fisheries.

Gibbs, R., Matthews, M., \& Link, D. (1971). The relationship between sphere size and settling velocity. Journal of Sedimentary Petrology, 41, 7-18.

Haley, S. R. (1969). Relative growth and sexual maturity of the Texas ghost crab, Ocypode quadrata (Fabr.) (Brachyura, Ocypodidae). Crustaceana, 17, 285-297.

Haley, S. R. (1972). Reproductive cycling in the ghost crab, Ocypode quadrata (Fabr.) (Brachyura, Ocypodidae). Crustaceana, 23, 1-11.

Herrmann, M., Carstensen, D., Fischer, S., Laudien, J., Penchaszadeh, P. E., \& Arntz, W. E. (2009). Population structure, growth and production of the wedge clam Donax hanleyanus (Bivalvia: Donacidae) from northern Argentinean beaches. Journal of Shellfish Research, 28, 511-526.

Herrera-Sansores, J. C. (2011). Clima. In C. Pozo, N. Armijo-Canto, \& S. Calmé (Eds.), Riqueza Biológica de Quintana Roo. Un análisis para su conservación (Tomo I, pp. 50-56). México, D. F., México: El Colegio de la Frontera Sur (Ecosur), Comisión Nacional para el Conocimiento y Uso de la Biodiversidad (Conabio), Gobierno del Estado de Quintana Roo y Programa de Pequeñas Donaciones (ppd).

Hobbs, C. H. III., Landry, C. B., \& Perry, J. E. III. (2008). Assessing anthropogenic and natural impacts on ghost crabs (Ocypode quadrata) at Cape Hatteras National Seashore, North Carolina. Journal of Coastal Research, 24, 1450-1458.

Hubbard, D. M., Dugan, J. E., Schooler, N. K., \& Viola, S. M. (2014). Local extirpations and regional declines of endemic upper beach invertebrates in southern California. Estuarine, Coastal and Shelf Science, 150, 67-75.

Leber, K. M. (1981). Spatial Patterns of Ocypode quadrata: A Re-Evaluation (Decapoda, Brachyura). Crustaceana, 41, 110-112.

Lucrezi, S., \& Schlacher, T. A. (2014). The ecology of ghost crabs. Oceanography and Marine Biology: An Annual Review, 52, 201-256.

Magalhães, W. F., Lima, J. B., Barros, F., \& Dominguez, J. M. L. (2009). Is Ocypode quadrata (Fabricius, 1787) a useful tool for exposed sandy beaches management in Bahia state (Northeast Brazil)? Brazilian Journal of Oceanography, 57, 149-152. 
McLachlan, A., \& Brown, A. C. (2006). The Ecology of Sandy Shores. Amsterdam, The Netherlands: Elsevier.

McLachlan, A., Defeo, O., Jaramillo, E., \& Short, A. D. (2013). Sandy beach conservation and recreation: Guidelines for optimising management strategies for multi-purpose use. Ocean \& Coastal Management, $71,256-268$.

Melo, G. A. S. (1996). Manual de identificação dos Brachyura (caranguejos e siris) do litoral brasileiro. São Paulo, Brasil: Plêiade.

Morrow, K., Bell, S. S., \& Tewfik, A. (2014). Variation in ghost crab trophic links on sandy beaches. Marine Ecology Progress Series, 502, 197-206.

Negreiros-Fransozo, M. L., Fransozo, A., \& Bertini, G. (2002). Reproductive cycle and recruitment period of Ocypode quadrata (Decapoda, Ocypodidae) at a sandy beach in southeastern Brazil. Journal of Crustacean Biology, 22, 157-161.

Nel, R., Campbell, E. E., Harris, L., Hauser, L., Schoeman, D. S., McLachlan, A., du Preez, D. R., Bezuidenhout, K., \& Schlacher, T. A. (2014). The status of sandy beach science: Past trends, progress, and possible futures. Estuarine, Coastal and Shelf Science, $150,1-10$.

Neves, F. M., \& Bemvenuti, C. E. (2006). The ghost crab Ocypode quadrata (Fabricius, 1787) as a potential indicator of anthropic impact along the Rio Grande do Sul coast, Brazil. Biological Conservation, 133, 431-435.

Noriega, R., Schlacher, T. A., \& Smeuninx, B. (2012). Reductions in Ghost Crab Populations Reflect Urbanization of Beaches and Dunes. Journal of Coastal Research, 28, 123-131.

Ocaña, F. A., Vega, A., \& Córdova, E. A. (2012). Distribución espacial de Ocypode quadrata (Decapoda: Ocypodidae) en ocho playas de la zona norte oriental de Cuba. Revista de Biología Tropical, 60, 1177-1186.

Pauly, D., \& Munro, J. L. (1984). Once more on the comparison of growth in fish and invertebrates. Fishbyte, $2,1-21$.

Petracco, M., Cardoso, R. S., \& Turra, A. (2013). Patterns of sandy-beach macrofauna production. Journal of the Marine Biological Association of the United Kingdom, 93, 1717-1725.

Pombo, M. (2015). The Atlantic ghost crab Ocypode quadrata (Decapoda: Ocypodidae) as bioindicator of sandy beaches: assessment of the influence of environmental, behavioral and population factors (Unpublished doctoral dissertation). Oceanographic Institute of the University of São Paulo.

Portell, R. W, Turner, R. L., \& Beerensson, J. L. (2003). Occurrence of the Atlantic Ghost Crab Ocypode quadrata from the Upper Pleistocene to Holocene Anastasia Formation of Florida. Journal of Crustacean Biology, 23, 712-722.

Robertson, J. R., \& Pfeiffer, W. J. (1982). Deposit-feeding by the ghost crab Ocypode quadrata (Fabricius). Journal of Experimental Marine Biology and Ecology, 56, 165-177.

Rosa, L. C., \& Borzone, C. A. (2008). Spatial distribution of Ocypode quadrata (Crustacea: Ocypodidae) along estuarine environments in the Paranaguá Bay Complex, Southern Brazil. Revista Brasileira de Zoologia, $25,383-388$.

Sakai, K., \& Türkay, M. (2013). Revision of the genus Ocypode with description of a new genus, Hoplocypode (Crustacea: Decapoda: Brachyura). Memoirs of the Queensland Museum, Nature, 56, 665-793.

Santos, M. C. F., \& Moreira, G. S. (1999). Time course of osmoionic compensations to acute salinity exposure in the ghost crab Ocypode quadrata (Fabricius, 1787). Journal of Experimental Marine Biology and Ecology, 235, 91-104.

Scapini, F. (2014). Behaviour of mobile macrofauna is a key factor in beach ecology as response to rapid environmental changes. Estuarine, Coastal and Shelf Science, 150, 36-44.

Schlacher, T. A., de Jager, R., \& Nielsen, T. (2011). Vegetation and ghost crabs in coastal dunes as indicators of putative stressors from tourism. Ecological Indicators, 11, 284-294.

Schlacher, T. A., Thompson, L., \& Price, S. (2007). Vehicles versus conservation of invertebrates on sandy beaches: mortalities inflicted by off-road vehicles on ghost crabs. Marine Ecology, 28, 354-367.

Short, A. (1996). The role of wave height, period, slope, tide range and embaymentisation in beach classifications: a review. Revista Chilena de Historia Natural, 69, 589-604.

Silva, W. T. A. F., \& Calado, T. C. S. (2013). Number of ghost crab burrows does not correspond to population size. Central European Journal of Biology, 8, 843-847.

Souza, J. R. B., Lavoie, N., Bonifácio, P. H., \& Rocha, C. M. C. (2008). Distribution of Ocypode quadrata (Fabricius, 1787) on sandy beaches of Northeastern Brazil. Atlântica, 30, 139-145.

Sparre, P., \& Venema, S. C. (1997). Introducción a la Evaluación de Recursos Pesqueros Tropicales Parte 1. Manual. Roma, Italia: FAO Fish Technical Papers 306.

Steiner, A. J., \& Leatherman, S. P. (1981). Recreational impacts on the distribution of ghost crab Ocypode quadrata Fab. Biological Conservation, 20, 111-122. 
Taylor, C. C. (1958). Cod growth and temperature. ICES Journal of Marine Science, 23, 366-370.

Tello-Taracena, H. A., \& Castellanos-Martínez E. O. (2011). Características geográficas. In C. Pozo, N. Armijo-Canto, \& S. Calmé (Eds.), Riqueza Biológica de Quintana Roo. Un análisis para su conservación (Tomo I, pp. 24-32). México, D. F., México: El Colegio de la Frontera Sur (Ecosur), Comisión Nacional para el Conocimiento y Uso de la Biodiversidad (Conabio), Gobierno del Estado de Quintana Roo y Programa de Pequeñas Donaciones (ppd).

Torres, G. M. (2010). Ecología de la taxocenósis de peracáridos macrobentónicos en playas arenosas protegidas: análisis multiescala de los patrones ecológoicos $y$ consideraciones para el manejo sustentable (Tesis de doctorado inédita). Centro de Investigaciones Biológicas del Noroeste S.C, La Paz, Baja California Sur.

Trott, T. J. (1998). On the sex ratio of the painted ghost crab Ocypode gaudichaudii H. Milne Edwards \& Lucas, 1843 (Brachyura, Ocypodidae). Crustaceana, $71,47-56$.

Tullis, A., \& Andrus, S. C. (2011). The cost of incline locomotion in ghost crabs (Ocypode quadrata) of different sizes. Journal of Comparative Physiology. B. Biochemical, Systemic, and Environmental Physiology, 181, 873-881.
Turra, A., Gonçalves, M. A. O., \& Denadai, M. R. (2005). Spatial distribution of the ghost crab Ocypode quadrata in low-energy tide-dominated sandy beaches. Journal of Natural History, 39, 2163-2177.

Valero-Pacheco, E., Álvarez, F., Abarca-Arenas, L. G., \& Escobar, M. (2007). Population density and activity pattern of the ghost crab, Ocypode quadrata, in Veracruz, Mexico. Crustaceana, 80, 313-325.

Vinagre, A. S., do Amaral, A. P. N., Ribarcki, F. P., da Silveira, E. F., \& Périco, E. (2007). Seasonal variation of energy metabolism in ghost crab Ocypode quadrata at Siriu Beach (Brazil). Comparative Biochemistry and Physiology A: Molecular and Integrative Physiology, 146, 514-519.

Williams, A. B. (1984). Shrimps, Lobsters and Crabs of the Atlantic Coast of the Eastern U.S. Maine to Florida. Washington, D.C., USA: Smithsonian Institution.

Wolcott, T. G. (1978). Ecological role of ghost crabs, Ocypode quadrata (Fab.) on an ocean beach: scavengers or predators? Journal of Experimental Marine Biology and Ecology, 31, 67-82.

Zar, J. H. (1999). Biostatistical Analysis. Upper Saddle River, USA: Prentice-Hall. 
